\title{
URETERO-VESICAL DYNAMICS IN RELATION TO REFLUX 1
}

\author{
By Herbert S. Talbot, M.D., Ass. Professor of Surgery, Harvard University, \\ Boston, U.S.A., Edward M. Mahoney, M.D., Julian E. Jarrett, M.D. \\ (Deceased), Oliver E. CobB, M.D.
}

STUDY of the function of the ureter must deal not only with its transporting urine from the kidney to the bladder, but also with the manner in which it prevents reversal of the normal flow. This includes prevention of vesico-ureteral reflux, and protection of the kidney from the high pressures generated in the bladder during micturition in normal subjects, and more or less constantly in certain states of dysfunction. Studies in this field have multiplied rapidly in recent years, and there is as yet no general agreement as to how these mechanisms operate. It is not likely that a single explanation can account for all cases of vesico-ureteral reflux. This study deals only with its occurrence in patients with neurogenic vesical dysfunction.

The ureter is fundamentally a simple organ with a simple task. It must get urine from the kidney to the bladder and prevent it from coming back. It has no other activities in the way of storage, secretion, or absorption. It would be reasonable to postulate, therefore, that no very elaborate apparatus is required for the regulation of its activities. The notion of its almost complete autonomy has recently been challenged by Boyarsky and his associates (1967), who present evidence of a functioning sympathetic nerve supply, but concede that its role requires further investigation. None the less, the ureter seems to have little dependence upon clinically observable autonomic factors. Its function, for instance, appears to show no alteration during the episodes of autonomic dysreflexia often observed after spinal cord injury. Neither does it respond to clinical efforts to control it with drugs, although such effects have been demonstrated experimentally. It does, however, depend strongly upon its own structural integrity.

The ureter in its ordinary activites encounters only two variables-(I) the amount of urine it must transport and (2) the pressure within the bladder against which it must move its burden. The rate at which the urine is delivered from the kidney is the most important determinant for the propulsive function, and the intravesical pressure for the mechanism to prevent backflow. The first affects the second only in so far as it causes the bladder to fill and empty more frequently. There is wide agreement that propulsive activity is mechanically stimulated by the presence of urine in the ureter. Prevention of backflow is dependent upon a valve-like action occurring at or near the uretero-vesical junction. There is still no clear anatomic evidence of the existence of an actual sphincter in this region. The valve effect has been explained by some as a passive occlusion, dependent upon the obliquity of the passage of the ureter through the bladder wall, the length of this passage, and the presence of a mucosal flap. Hutch (1958) amplifies this by the addition of a dynamic concept, 'The intravesical ureter plays an active part in the prevention of reflux. At the conclusion

${ }^{1}$ From the Spinal Cord Injury Service and the Urology Section of the Surgical Service, Veterans Administration Hospital, West Roxbury, Massachusetts. 
of the spurt of urine, the longitudinal muscles of the ureter occlude the lumen of the ureter so that no bladder contents may enter'. He thus attributes the development of reflux to alterations in the juxta-ureteral bladder wall, congenital or acquired, which might impede this mechanism.

There is no reason to doubt that these factors play a part, and failure of any one may predispose to failure of the entire mechanism in a given instance. But this does not exclude the probability of a more intricate mechanism, the existence of which is suggested by a number of facts. Taken together, they suggest that the lower ureteral segment constitutes a functional ureterovesical valve, this segment including not only the intravesical portion, but perhaps the entire lower third. Anatomically, the wall here is the thickest found in any portion of the ureter, and its outer muscular layer merges indistinguishably with the musculature of the bladder. The lower third of the ureter, moreover, is supplied with parasympathetic innervation similar to that of the detrusor (Torkey \& Leadbetter, I963). Thus, there is strong anatomic presumption of an intimate functional relationship between the bladder and the lower ureteral segment.

Gould, Hsieh, and Tinckler (1955) have suggested that the lower end of the ureter has a task somewhat different from that of the upper, and that the integration of tonic and peristaltic activity is such as normally to protect the nephron from the high basic pressures which arise in the lower segment when it is operating to handle large quantities of urine, or against elevated intravesical pressures.

Fluoroscopic studies, including cinefluorographic investigations, have demonstrated that the lower ureter does, in fact, contract during or immediately after an emptying contraction of the bladder. Again, fluoroscopically, it has been possible to demonstrate that in some patients with vesico-ureteral reflux the mechanism remains sufficiently well compensated to operate in approximately normal fashion, and in such instances the kidney may remain undamaged for months or even years (Talbot, I958). When alterations become sufficiently damaging, however, decompensation occurs and drainage becomes impaired. Even before anatomic hydronephrosis can be demonstrated, pressure changes, damaging to renal function and conducive to infection, may already be present. Reverse peristalsis, long since observed in the presence of mechanical obstruction, may occur also in this functional type.

Previous investigations have suggested cessation of ureteral peristalsis during micturition. Kiil (1957) was unable to confirm this and found no reflexogenic regulation of ureteral activity during bladder filling and micturition. He did observe, however, that a prolonged large increase in intravesical pressure led to an increase in ureteral resting pressure and, in turn, to an increased frequency of ureteral contraction; this occurs also during a high rate of urine production. Lapides (1948) and others have shown that, in general, the lower segment maintains a higher degree of tonus and contracts more vigorously than the upper. Gould and his associates (1955) found in the suffused isolated ureter that 'tone increased from above downwards, and that autonomic drugs exert their maximum effect upon tone through the muscle of the lower end'. 'The ureter', they point out, 'may continue to force urine into the bladder during micturition, but it is unlikely that an elaborate mechanism exists solely for ensuring the onward passage of urine during so brief and occasional an event.' 
There is, however, a mechanism which serves to prevent the backflow of urine into the ureter when the bladder pressure rises-in fact, a dynamic uretero-vesical valve. Whether this depends upon neural controls or is activated simply by the mechanical stimulus applied through a local distension of smooth muscle is not yet known, but in either event its effective working requires a structurally sound ureteral wall. It is significant that when reflux first develops it is often associated with dilatation of the lower segment, which is sometimes the first sign of its presence (Talbot \& Bunts, 1949). But since the lower segment possesses the thickest and strongest muscular wall, it should not be the first to show dilatation under the pressure of flow from the kidney with obstruction occurring distally. Alteration in the ureter itself-usually caused by infection spreading from the contiguous bladder wall-prevents its normal contraction, and this results in reflux and functional obstruction, the lumen remaining quite patent.

The association of uretero-vesical reflux with infection has had increasing attention during recent years. It has been suggested that pyelonephritis may, in fact, cause reflux (Kaveggia et al., 1966). Such a situation, however, is obviously less frequent than that in which the primary involvement is in the lower tract. Sommer and Roberts (1966) have reported increased incidence of reflux in dogs with chronic urinary tract infection. Lich and his associates (1964) found reflux among new-born infants only when infection was present, and quote Tanagho's observation to the same effect. Hackler, Dalton and Bunts (1965) consider infection to be particularly significant in patients with neurogenic bladders. A classical experiment has been described by Schoenberg et al. (1964). They produced persistent infection of the bladder wall in dogs by an ingenious method which obviated use of a foreign body. In 9 of 22 animals so treated, reflux developed without prior detectable alteration of hydrodynamics. It never appeared in animals that failed to develop persistent infection, nor in those subjected to a sham operation.

In the earlier stages of the dysfunction, reflux may be unaccompanied by any other notable change in ureteral activity. As infection spreads upward in the ureteral wall, however, there is increasing impairment of peristalsis, culminating in its complete cessation. Grand et al. (1965) found this both clinically and experimentally. In the series of patients here reported, this variable degree of dysfunction associated with reflux was repeatedly demonstrated and, when specimens were obtainable, related to microscopic as well as gross changes in the ureter. Vesico-ureteral reflux, therefore, cannot be considered as a sole cause of subsequent hydronephrosis. The association is common and has been well documented by Hutch, Hinman and Miller (1962), among others. In the cases here reported, it is more properly to be regarded as one factor-a very important one-in a series of dysfunctions, initiated by infection in the ureteral wall.

The importance of the lower segment of the ureter cannot be disregarded. Scott (I964) performed an experiment designed to demonstrate the effects of prolonged vesico-ureteral reflux. He concluded that it was not possible to state that reflux predisposes to urinary infection. It did cause alteration in the behaviour of peristalsis and deterioration in the function of the upper tract. He produced reflux in dogs by excision of the mucosal flap. In the acute experiments, it did not develop during life, but was evident after the animals had been killed and peristalsis thus abolished. Torkey and Leadbetter, already quoted, point out that the parasympathetic supply to the lower ureter definitely exerts a stimulating 
effect on peristalsis and perhaps helps to prevent reflux during bladder contraction. The accumulation of experimental and clinical experience leaves no reasonable doubt that the contraction of the lower ureteral segment plays an important part in the valve-like function of the uretero-vesical junction.

It is unwarranted, however, to attempt to postulate the same etiology as being operative in every instance. Dysfunction might result from any alteration, acquired or congenital, which affected the normal contractility of the lower ureteral segment or the structure of the uretero-vesical junction. If it could be demonsstrated, for instance, that neural control played a part, absence of or interference

\section{TABLE I}

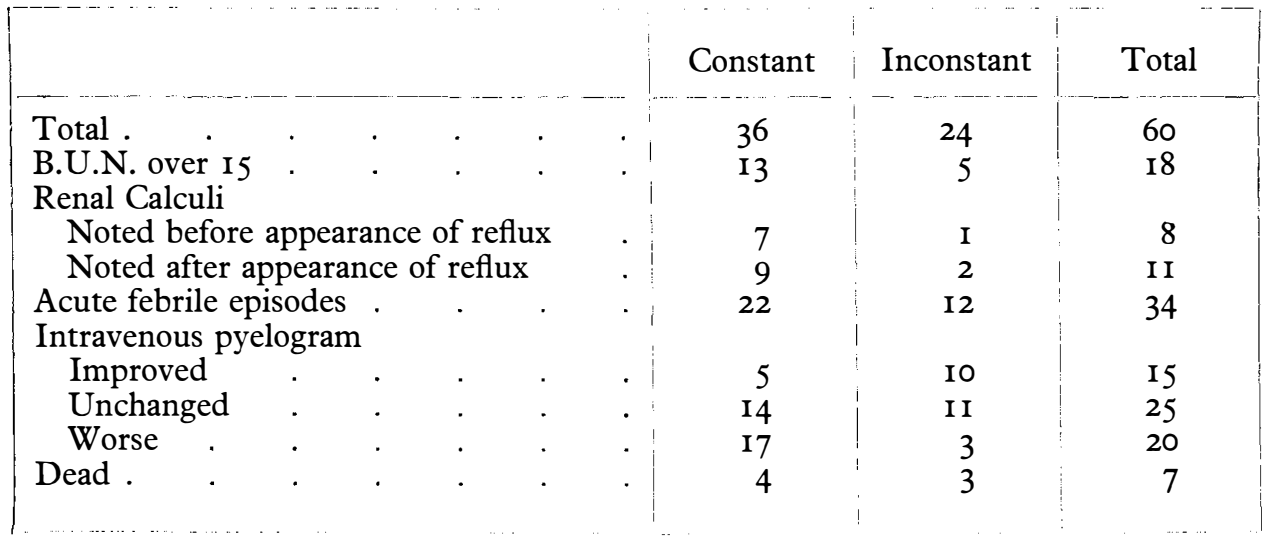

with the nerve supply might be the cause. The several etiologies that have been suggested are not mutually exclusive and may, on occasion, operate together.

Some clinical implications of the uretero-vesical relationship emerge from a study of 60 patients with vesico-ureteral reflux observed for periods of I to I5 years, except for one who died within a year. This comprised all patients with reflux seen during the period of study; all but 2 had been re-examined within six months of its completion.

Reflux represents a significant derangement, whatever its cause, of the reciprocal vesico-ureteral function. It may not, however, be constantly evident in every case (Bors \& Comarr, 1952). In this series, there were 36 patients in whom reflux was noted at every examination. In 24 , it was inconstant. Rarely, it disappeared after a single demonstration; more often it was manifest on several occasions and then was seen no more, although commonly associated signs, such as trabeculation of the bladder and dilatation of the lower ureter, might persist. In a few cases its appearance has been intermittent. Such inconstancy is understandable in terms of the theory that reflux may be due to inflammatory changes in the wall of the lower ureter, but it makes classification and tabulation difficult and confusing. Since cystograms are not ordinarily made at frequent intervals, it cannot be determined accurately just how long reflux might have been present between an examination that showed it and one that did not. Thus no clear differentiation between the constant and inconstant groups is possible in terms of the actual duration of reflux. 
The division into the constant and inconstant was at first adopted only tentatively as a convenient starting point, but when the data were tabulated, the two groups showed interesting clinical differences (Table I). It might, of course, have been expected that a persistent would be more damaging than an intermittent or transitory dysfunction, but it was not expected that the differences would be so evident. To begin with the least striking, 6I per cent. of the constant group

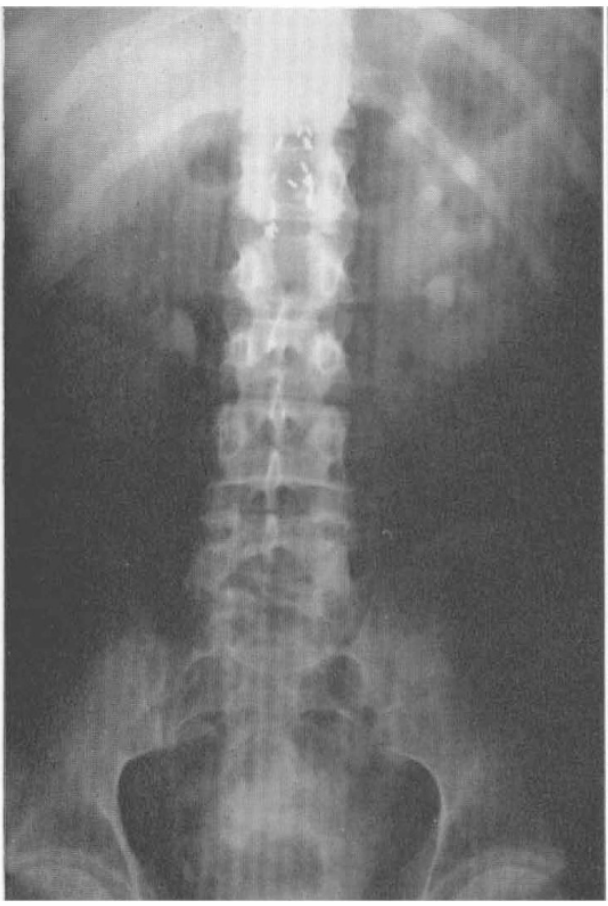

A

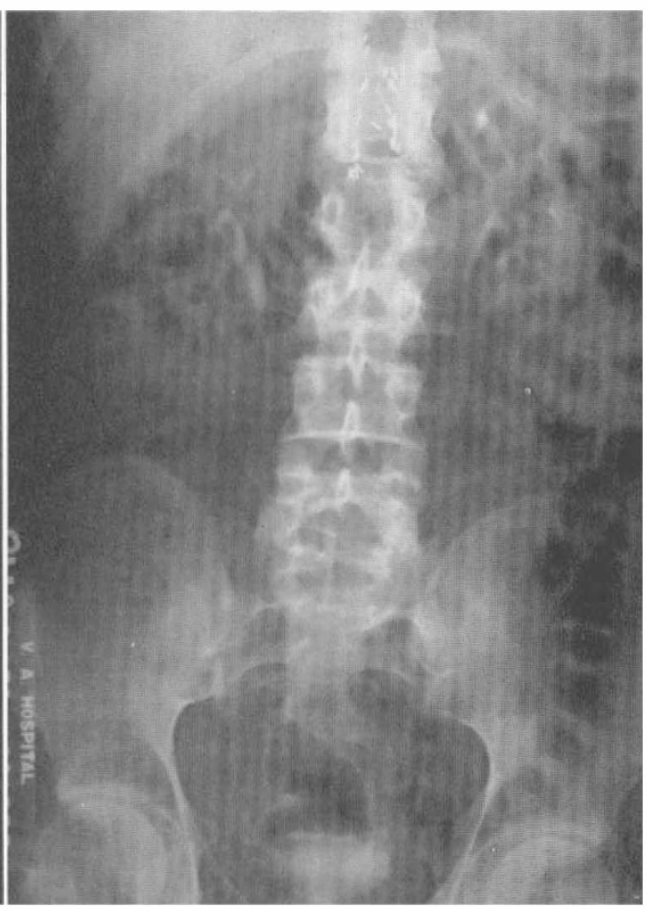

B

FIG. I

A, Left hydronephrosis. Constant reflux on cystogram. Patient placed on permanent catheter drainage.

B, Regression of hydronephrosis 5 years later. Reflux no longer present.

had had, during the period of observation, more than one acute febrile episode attributed to renal infection; in the inconstant group the figure was 50 per cent. Considering the small size of the series, the difference is probably not significant. But this is, as a matter of fact, a particularly difficult factor to establish. Among patients with spinal cord injury or disease, in whom pain and tenderness over the kidney were usually absent, and whose urine almost always contained white blood cells and bacteria, almost any febrile episode was likely to have been considered pyelonephritis until proved otherwise.

Renal calculi developed in 42 per cent. of patients in the constant group, in only $12 \frac{1}{2}$ per cent. of the others. Levels of blood urea nitrogen exceeding I5 mg. per cent. were found (at the most recent examination) in approximately 36 per cent. and 20 per cent. of the two groups, respectively. There were four 
deaths among the patients who showed reflux on every examination, three of which were due to renal failure, a mortality of about 8 per cent. from this cause. Among the others there were three deaths, all from amyloid disease.

Apart from the mortality, the most striking findings are related to changes in the ureters and kidneys as revealed in intravenous pyelograms taken at regular intervals throughout the period of observation. Improvement was evident in 4I per cent. of the inconstant group but only about I4 per cent. of the constant (figs. IA and B and $2 \mathrm{~A}$ and B). On the other hand, one-half of the constant group

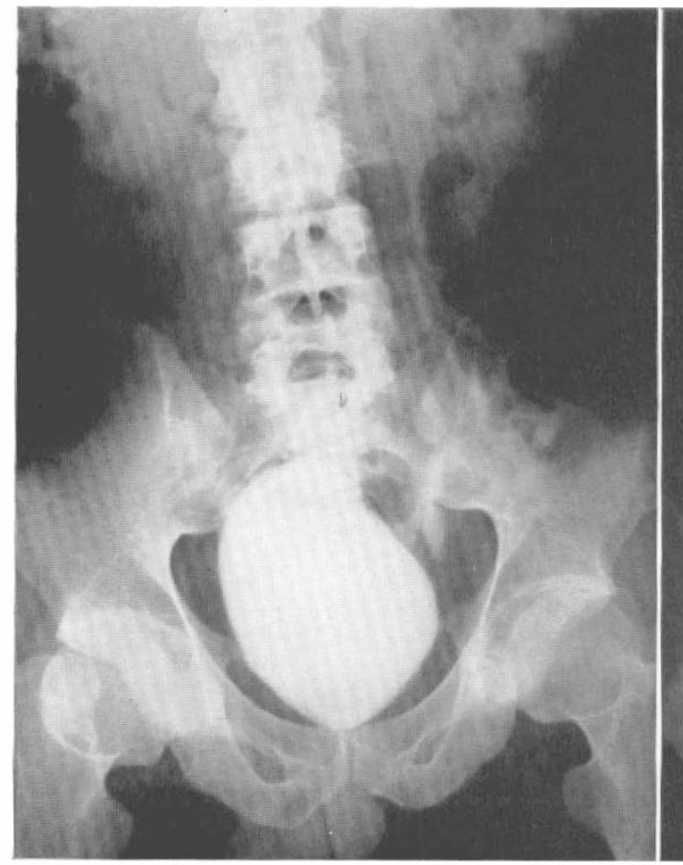

A

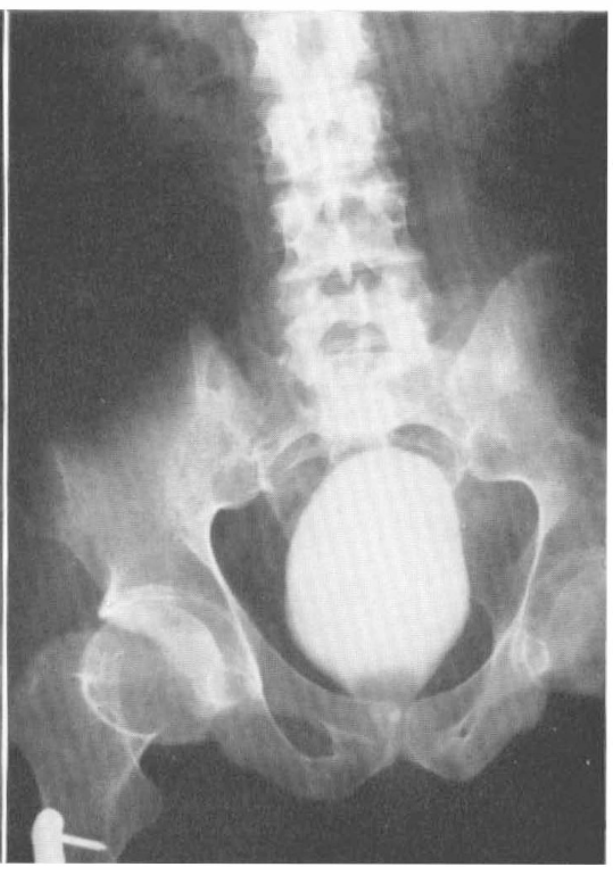

B

FIG. 2

A, Left vesico-ureteral reflux, constant, of Io years' duration.

B, Disappearance of reflux after 3 months of catheter drainage during treatment for a fractured femur.

showed deterioration, while this occurred in only one-eighth of the inconstant (figs. 3A and B). Once developed, such changes are usually progressive, but even a well-marked hydronephrosis may prove to be reversible, although reflux may remain. The determining factor, as suggested by fluoroscopic observation, is the dynamic capacity of the ureter, rather than simply its structural appearance. Since reflux may disappear spontaneously, evaluation of treatment by this finding alone must be cautious. Observations on the progress or regression of changes in the upper tract are more important, and these are the best criteria upon which to base treatment. 


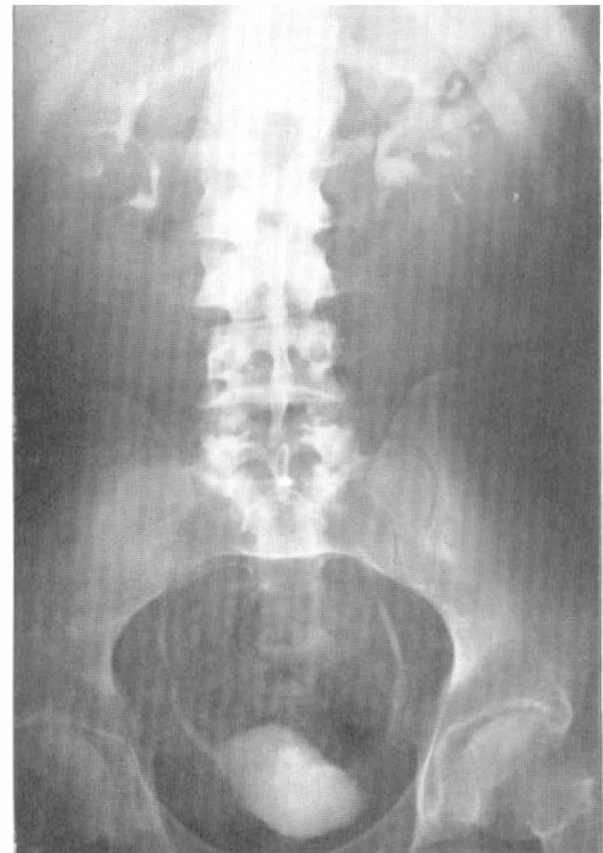

A

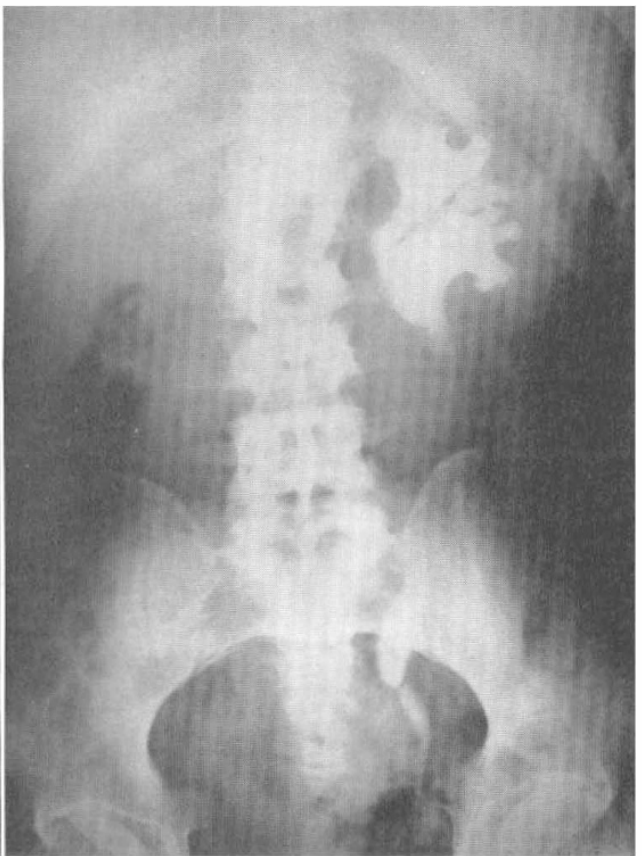

B

FIG. 3

A, Intravenous pyelogram of patient with constant bilateral reflux, without significant change in upper tract. Patient voiding.

B, Deterioration Io months later; hydro-ureter and hydronephrosis, left; diminished function, right.

\section{REFERENCES}

Bors, E. \& Comarr, A. E. (I952). F. Urol. 68, 68I.

Boyarsky, S., LABAy, P., KirschNeR, N. \& Gerber, C. (I967). f. Urol. 97, 627.

Gould, D. W., Hsieh, A. C. L. \& Tinckler, L. F. (I955). F. Physiol. 29, 436.

Grand, L., KIDD, J., IdRISS, F. \& Swenson, O. (I965). F. Urol. 94, 652.

HACKLeR, R. H., Dalton, J. J. \& Bunts, R. C. (I965). F. Urol. 94, IO7.

HuTCH, J. A. (1958). Uretero-vesical function; the theory of extra-vesicalization of the intra-vesical ureter. University of California Press.

Hutch, J. A., Hinman, F., Jr. \& MilleR, E. R. (I962). F. Urol., 88, I69.

KIIL, F. (I957). The Function of the Ureter and Renal Pelvis. Philadelphia: W. B. Saunders Co.

KavegGia, L., King, L. R., Grana, L. \& Idriss, F. S. (I966). F. Urol. 95, I58.

LAPIDES, J. (I948). F. Urol. 59, 50I.

Lich, R., Jr., Howerton, L. W., Jr., Goode, L. S. \& Davis, L. A. (I964). F. Urol. 92, 436.

Schoenderg, H. W., Beisswanger, P., Howard, W. J., Klingenmaier, H., Walter, C. F. \& MURPHY, J. J. (1964). F. Urol. 92, 107.

ScotT, J. E. S. (1964). Br. F. Urol., 36, 391.

SOMMER, J. L. \& ROBERTS, J. A. (I966). F. Urol. 95, 502.

Talbot, H. S. (1958). F. Am. med. Ass. I68, I595.

TAlbot, H. S. \& BUNTs, R. C. (I949). F. Urol. 6I, 870.

TANagho, E. A. \& Hutch, J. A. (1965). F. Urol., 93, I 58.

TORKEY, K. \& LeadBETTER, W. F. (I963). F. Urol. 90, 395. 